\title{
Approximate Solutions of Nonlinear Partial Differential Equations by Modified $q$-Homotopy Analysis Method
}

\author{
Shaheed N. Huseen ${ }^{1}$ and Said R. Grace ${ }^{2}$ \\ ${ }^{1}$ Mathematics Department, Faculty of Computer Science and Mathematics, Thi-Qar University, Thi-Qar, Iraq \\ ${ }^{2}$ Engineering Mathematics Department, Faculty of Engineering, Cairo University, Giza, Egypt
}

Correspondence should be addressed to Shaheed N. Huseen; shn_n2002@yahoo.com

Received 27 May 2013; Accepted 23 September 2013

Academic Editor: Alexander Timokha

Copyright (C) 2013 S. N. Huseen and S. R. Grace. This is an open access article distributed under the Creative Commons Attribution License, which permits unrestricted use, distribution, and reproduction in any medium, provided the original work is properly cited.

\begin{abstract}
A modified $q$-homotopy analysis method (mq-HAM) was proposed for solving $n$ th-order nonlinear differential equations. This method improves the convergence of the series solution in the $n$ HAM which was proposed in (see Hassan and El-Tawil 2011, 2012). The proposed method provides an approximate solution by rewriting the $n$ th-order nonlinear differential equation in the form of $n$ first-order differential equations. The solution of these $n$ differential equations is obtained as a power series solution. This scheme is tested on two nonlinear exactly solvable differential equations. The results demonstrate the reliability and efficiency of the algorithm developed.
\end{abstract}

\section{Introduction}

Homotopy analysis method (HAM) initially proposed by Liao in his Ph.D. thesis [1] is a powerful method to solve nonlinear problems. In recent years, this method has been successfully employed to solve many types of nonlinear problems in science and engineering [2-17]. HAM contains a certain auxiliary parameter $h$, which provides us with a simple way to adjust and control the convergence region and rate of convergence of the series solution. Moreover, by means of the so-called $h$-curve, a valid region of $h$ can be studied to gain a convergent series solution. More recently, a powerful modification of HAM was proposed [18-20]. Hassan and ElTawil $[21,22]$ presented a new technique of using homotopy analysis method for solving nonlinear initial value problems $(n \mathrm{HAM})$. El-Tawil and Huseen $[23,24]$ established a method, namely, $q$-homotopy analysis method, ( $q$-HAM) which is a more general method of HAM, The $q$-HAM contains an auxiliary parameter $n$ as well as $h$ such that the case of $n=$ 1 ( $q$-HAM; $n=1)$ and the standard homotopy analysis method (HAM) can be reached. In this paper, we present the modification of $q$-homotopy analysis method (mq-HAM) for solving nonlinear problems by transforming the $n$ th-order nonlinear differential equation to a system of $n$ first-order equations. we note that the $n \mathrm{HAM}$ is a special case of $\mathrm{m} q$ $\operatorname{HAM}(\mathrm{m} q-\mathrm{HAM} ; n=1)$.

\section{Analysis of the $q$-Homotopy Analysis Method ( $q$-HAM)}

Consider the following nonlinear partial differential equation:

$$
N[u(x, t)]=0,
$$

where $N$ is a nonlinear operator, $(x, t)$ denotes independent variables, and $u(x, t)$ is an unknown function. Let us construct the so-called zero-order deformation equation as follows:

$$
(1-n q) L\left[\emptyset(x, t ; q)-u_{0}(x, t)\right]=q h H(x, t) N[\emptyset(x, t ; q)]
$$

where $n \geq 1, q \in[0,1 / n]$ denotes the so-called embedded parameter, $L$ is an auxiliary linear operator with the property $L[f]=0$ when $f=0, h \neq 0$ is an auxiliary parameter, and 
$H(x, t)$ denotes a non-zero auxiliary function. It is obvious that when $q=0$ and $q=1 / n$, (2) becomes

$$
\emptyset(x, t ; 0)=u_{0}(x, t), \quad \emptyset\left(x, t ; \frac{1}{n}\right)=u(x, t),
$$

respectively. Thus, as $q$ increases from 0 to $1 / n$, the solution $\emptyset(x, t ; q)$ varies from the initial guess $u_{0}(x, t)$ to the solution $u(x, t)$. We may choose $u_{0}(x, t), L, h$, and $H(x, t)$ and assume that all of them can be properly chosen so that the solution $\emptyset(x, t ; q)$ of $(2)$ exists for $q \in[0,1 / n]$.

Now, by expanding $\emptyset(x, t ; q)$ in Taylor series, we have

$$
\emptyset(x, t ; q)=u_{0}(x, t)+\sum_{m=1}^{+\infty} u_{m}(x, t) q^{m}
$$

where

$$
u_{m}(x, t)=\left.\frac{1}{m !} \frac{\partial^{m} \emptyset(x, t ; q)}{\partial q^{m}}\right|_{q=0}
$$

Next, we assume that $h, H(x, t), u_{0}(x, t)$, and $L$ are properly chosen such that the series (4) converges at $q=1 / n$ and that

$$
u(x, t)=\emptyset\left(x, t ; \frac{1}{n}\right)=u_{0}(x, t)+\sum_{m=1}^{+\infty} u_{m}(x, t)\left(\frac{1}{n}\right)^{m} .
$$

Let

$$
u_{r}(x, t)=\left\{u_{0}(x, t), u_{1}(x, t), u_{2}(x, t), \ldots, u_{r}(x, t)\right\} .
$$

Differentiating equation (2) for $m$ times with respect to $q$ and then setting $q=0$ and dividing the resulting equation by $m$ !, we have the so-called $m$ th order deformation equation as follows:

$$
L\left[u_{m}(x, t)-k_{m} u_{m-1}(x, t)\right]=h H(x, t) R_{m}\left(\overrightarrow{u_{m-1}}(x, t)\right),
$$

where

$$
\begin{gathered}
R_{m}\left(\overrightarrow{u_{m-1}}(x, t)\right) \\
=\left.\frac{1}{(m-1) !} \frac{\partial^{m-1}(N[\emptyset(x, t ; q)]-f(x, t))}{\partial q^{m-1}}\right|_{q=0}, \\
k_{m}= \begin{cases}0 & m \leq 1, \\
n & \text { otherwise. }\end{cases}
\end{gathered}
$$

It should be emphasized that $u_{m}(x, t)$ for $m \geq 1$ is governed by the linear equation (8) with linear boundary conditions that come from the original problem. Due to the existence of the factor $(1 / n)^{m}$, more chances for convergence may occur or even much faster convergence can be obtained better than the standard HAM. It should be noted that the case of $n=1$ in (2), standard HAM, can be reached.

The $q$-homotopy analysis method ( $q$-HAM) can be reformatted as follows.
We rewrite the nonlinear partial differential equation (1) in the following form:

$$
\begin{gathered}
L u(x, t)+A u(x, t)+B u(x, t)=0, \\
u(x, 0)=f_{0}(x), \\
\left.\frac{\partial u(x, t)}{\partial t}\right|_{t=0}=f_{1}(x), \\
\vdots \\
\left.\frac{\partial^{z-1} u(x, t)}{\partial^{z-1}}\right|_{t=0}=f_{z-1}(x),
\end{gathered}
$$

where $L=\partial^{z} / \partial t^{z}, z=1,2, \ldots$ is the highest partial derivative with respect to $t, A$ is a linear term, and $B$ is a nonlinear term. The so-called zero-order deformation equation (2) becomes

$$
\begin{aligned}
& (1-n q) L\left[\emptyset(x, t ; q)-u_{0}(x, t)\right] \\
& =q h H(x, t)(L u(x, t)+A u(x, t)+B u(x, t)),
\end{aligned}
$$

we have the following $m$ th order deformation equation:

$$
\begin{aligned}
L & {\left[u_{m}(x, t)-k_{m} u_{m-1}(x, t)\right] } \\
& =h H(x, t)\left(L u_{m-1}(x, t)+A u_{m-1}(x, t)+B\left(\overrightarrow{u_{m-1}}(x, t)\right)\right) .
\end{aligned}
$$

Hence,

$$
\begin{aligned}
u_{m}(x, t)= & k_{m} u_{m-1}(x, t) \\
+ & h L^{-1}\left[H ( x , t ) \left(L u_{m-1}(x, t)+A u_{m-1}(x, t)\right.\right. \\
& \left.\left.+B\left(\overrightarrow{u_{m-1}}(x, t)\right)\right)\right] .
\end{aligned}
$$

Now, the inverse operator $L^{-1}$ is an integral operator which is given by

$$
L^{-1}(\cdot)=\iint \cdots \int(\cdot) \frac{d t d t \cdots d t}{z \text { times }}+c_{1} t^{z-1}+c_{2} t^{z-2}+\cdots+c_{z},
$$

where $c_{1}, c_{2}, \ldots, c_{z}$ are integral constants.

To solve (10) by means of $q$-HAM, we choose the following initial approximation:

$$
\begin{aligned}
u_{0}(x, t)= & f_{0}(x)+f_{1}(x) t \\
& +f_{2}(x) \frac{t^{2}}{2 !}+\cdots+f_{z-1}(x) \frac{t^{z-1}}{(z-1) !} .
\end{aligned}
$$

Let $H(x, t)=1$, by means of (14) and (15); then (13) becomes

$$
\begin{aligned}
& u_{m}(x, t) \\
& =k_{m} u_{m-1}(x, t) \\
& +h \int_{0}^{t} \int_{0}^{t} \cdots \int_{0}^{t}\left(\frac{\partial^{z} u_{m-1}(x, \tau)}{\partial \tau^{z}}+A u_{m-1}(x, \tau)\right. \\
& \left.+B\left(\overline{u_{m-1}}(x, \tau)\right)\right) \frac{d \tau d \tau \cdots d \tau .}{z \text { times }} .
\end{aligned}
$$


Now from $\int_{0}^{t} \int_{0}^{t} \cdots \int_{0}^{t}\left(\partial^{z} u_{m-1}(x, \tau) / \partial \tau^{z}\right) \underbrace{d \tau d \tau \cdots d \tau}_{z \text { times }}$, we observe that there are repeated computations in each step which caused more consuming time. To cancel this, we use the following modification to (16):

$$
\begin{aligned}
& u_{m}(x, t) \\
& =k_{m} u_{m-1}(x, t) \\
& +h \int_{0}^{t} \int_{0}^{t} \cdots \int_{0}^{t} \frac{\partial^{z} u_{m-1}(x, \tau)}{\partial \tau^{z}} \underbrace{d \tau d \tau \cdots d \tau}_{z \text { times }} \\
& +h \int_{0}^{t} \int_{0}^{t} \cdots \int_{0}^{t}\left(A u_{m-1}(x, \tau)\right. \\
& \left.+B\left(\overrightarrow{u_{m-1}}(x, \tau)\right)\right) \underbrace{d \tau d \tau \cdots d \tau}_{z \text { times }} \\
& =k_{m} u_{m-1}(x, t)+h u_{m-1}(x, t) \\
& -h\left(u_{m-1}(x, 0)+t \frac{\partial u_{m-1}(x, 0)}{\partial t}\right. \\
& \left.+\cdots+\frac{t^{z-1}}{(z-1) !} \frac{\partial^{z-1} u_{m-1}(x, 0)}{\partial t^{z-1}}\right) \\
& +h \int_{0}^{t} \int_{0}^{t} \cdots \int_{0}^{t}\left(A u_{m-1}(x, \tau)\right. \\
& \left.+B\left(\overrightarrow{u_{m-1}}(x, \tau)\right)\right) \underbrace{d \tau d \tau \cdots d \tau}_{z \text { times }} \text {. }
\end{aligned}
$$

Now, for $m=1, k_{m}=0$, and

$$
\begin{gathered}
u_{0}(x, 0)+t \frac{\partial u_{0}(x, 0)}{\partial t}+\frac{t^{2}}{2 !} \frac{\partial^{2} u_{0}(x, 0)}{\partial t^{2}} \\
+\cdots+\frac{t^{z-1}}{(z-1) !} \frac{\partial^{z-1} u_{0}(x, 0)}{\partial t^{z-1}} \\
=f_{0}(x)+f_{1}(x) t+f_{2}(x) \frac{t^{2}}{2 !} \\
+\cdots+f_{z-1}(x) \frac{t^{z-1}}{(z-1) !} \\
=u_{0}(x, t) .
\end{gathered}
$$

Substituting this equality into (17), we obtain

$$
\begin{aligned}
& u_{1}(x, t) \\
& \quad=h \int_{0}^{t} \int_{0}^{t} \cdots \int_{0}^{t}\left(A u_{0}(x, \tau)+B\left(u_{0}(x, \tau)\right)\right) \underbrace{d \tau d \tau \cdots d \tau .}_{z \text { times }}
\end{aligned}
$$

For $m>1, k_{m}=n$, and

$$
\begin{gathered}
u_{m}(x, 0)=0, \quad \frac{\partial u_{m}(x, 0)}{\partial t}=0, \\
\frac{\partial^{2} u_{m}(x, 0)}{\partial t^{2}}=0, \ldots, \frac{\partial^{z-1} u_{m}(x, 0)}{\partial t^{z-1}}=0 .
\end{gathered}
$$

Substituting this equality into (17), we obtain

$$
\begin{aligned}
& u_{m}(x, t) \\
& =(n+h) u_{m-1}(x, t) \\
& +h \int_{0}^{t} \int_{0}^{t} \cdots \int_{0}^{t}\left(A u_{m-1}(x, \tau)\right. \\
& \left.+B\left(\overline{u_{m-1}}(x, \tau)\right)\right) \frac{d \tau d \tau \cdots d \tau .}{z \text { times }} .
\end{aligned}
$$

The standard $q$-HAM is powerful when $z=1$, and the series solution expression by $q$-HAM can be written in the following form:

$$
u(x, t ; n ; h) \cong U_{M}(x, t ; n ; h)=\sum_{i=0}^{M} u_{i}(x, t ; n ; h)\left(\frac{1}{n}\right)^{i} .
$$

But when $z \geq 2$, there are too many additional terms where harder and more time consuming computations are performed. So, the closed form solution needs more numbers of iteration.

\section{The Proposed Modified $q$-Homotopy Analysis Method (mq-HAM)}

When $z \geq 2$, we rewrite (1) as in the following system of firstorder differential equations:

$$
\begin{gathered}
u_{t}=u 1, \\
u 1_{t}=u 2, \\
\vdots \\
u\{z-1\}_{t}=-A u(x, t)-B u(x, t) .
\end{gathered}
$$

Set the initial approximation

$$
\begin{aligned}
u_{0}(x, t) & =f_{0}(x), \\
u 1_{0}(x, t) & =f_{1}(x), \\
& \vdots \\
u\{z-1\}_{0}(x, t) & =f_{z-1}(x) .
\end{aligned}
$$

Using the iteration formulas (19) and (21) as follows:

$$
\begin{array}{r}
u_{1}(x, t)=h \int_{0}^{t}\left(-u 1_{0}(x, \tau)\right) d \tau, \\
u 1_{1}(x, t)=h \int_{0}^{t}\left(-u 2_{0}(x, \tau)\right) d \tau,
\end{array}
$$

$$
u\{z-1\}_{1}(x, t)=h \int_{0}^{t}\left(A u_{0}(x, \tau)+B\left(u_{0}(x, \tau)\right)\right) d \tau
$$


For $m>1, k_{m}=n$, and

$$
\begin{gathered}
u_{m}(x, 0)=0, \quad u 1_{m}(x, 0)=0, \\
u 2_{m}(x, 0)=0, \ldots, u\{z-1\}_{m}(x, 0)=0 .
\end{gathered}
$$

Substituting in (17), we obtain

$$
\begin{aligned}
& u_{m}(x, t)=(n+h) u_{m-1}(x, t)+h \int_{0}^{t}\left(-u 1_{m-1}(x, \tau)\right) d \tau, \\
& u 1_{m}(x, t)=(n+h) u 1_{m-1}(x, t) \\
& +h \int_{0}^{t}\left(-u 2_{m-1}(x, \tau)\right) d \tau, \\
& u\{z-1\}_{m}(x, t) \\
& =(n+h) u\{z-1\}_{m-1}(x, t) \\
& +h \int_{0}^{t}\left(A u_{m-1}(x, \tau)+B\left(u_{m-1}(x, \tau)\right)\right) d \tau .
\end{aligned}
$$

It should be noted that the case of $n=1$ in (27), the $n$ HAM, can be reached.

To illustrate the effectiveness of the proposed mq-HAM, comparison between $\mathrm{m} q$-HAM and the $n \mathrm{HAM}$ are illustrated by the following examples.

\section{Illustrative Examples}

Example 1. Consider the following nonlinear sine-Gordon equation:

$$
u_{t t}-u_{x x}+\sin u=0
$$

subject to the following initial conditions:

$$
u(x, 0)=0, \quad u_{t}(x, 0)=4 \operatorname{sech} x .
$$

The exact solution is

$$
u(x, t)=4 \tan ^{-1}(t \operatorname{sech} x) .
$$

In order to prevent suffering from the strongly nonlinear term $\sin u$ in the frame of $q$-HAM, we can use Taylor series expansion of $\sin u$ as follows:

$$
\sin u=u-\frac{u^{3}}{6}+\frac{u^{5}}{120}
$$

Then, (28) becomes

$$
u_{t t}-u_{x x}+u-\frac{u^{3}}{6}+\frac{u^{5}}{120}=0 .
$$

In order to solve (28) by mq-HAM, we construct system of differential equations as follows:

$$
\begin{gathered}
u_{t}(x, t)=v(x, t), \\
v_{t}(x, t)=\frac{\partial^{2} u(x, t)}{\partial x^{2}}-u+\frac{u^{3}}{6}-\frac{u^{5}}{120},
\end{gathered}
$$

with the following initial approximations:

$$
u_{0}(x, t)=0, \quad v_{0}(x, t)=4 \operatorname{sech} x,
$$

and the following auxiliary linear operators:

$$
\begin{aligned}
L u(x, t) & =\frac{\partial u(x, t)}{\partial t}, \quad L v(x, t)=\frac{\partial v(x, t)}{\partial t} \\
A u_{m-1}(x, t)=-\frac{\partial^{2} u_{m-1}(x, t)}{\partial x^{2}}+u_{m-1}(x, t) & \\
B \overline{u_{m-1}}(x, t)= & -\frac{1}{6} \sum_{j=0}^{m-1} u_{m-1-j} \sum_{i=0}^{j} u_{i} u_{j-i} \\
& +\frac{1}{120} \sum_{j=0}^{m-1} u_{m-1-j} \sum_{i=0}^{j} u_{j-i} \sum_{k=0}^{i} u_{i-k} \sum_{l=0}^{k} u_{l} u_{k-l}
\end{aligned}
$$

From (25) and (27), we obtain

$$
\begin{gathered}
u_{1}(x, t)=h \int_{0}^{t}\left(-v_{0}(x, \tau)\right) d \tau \\
v_{1}(x, t)=h \int_{0}^{t}\left(-\frac{\partial^{2} u_{0}}{\partial x^{2}}+u_{0}-\frac{u_{0}^{3}}{6}+\frac{u_{0}^{5}}{120}\right) d \tau .
\end{gathered}
$$

Now, for $m \geq 2$, we get

$$
\begin{aligned}
u_{m}(x, t)= & (n+h) u_{m-1}(x, t)+h \int_{0}^{t}\left(-v_{m-1}(x, \tau)\right) d \tau \\
v_{m}(x, t)= & (n+h) v_{m-1}(x, t) \\
& +h \int_{0}^{t}\left(A u_{m-1}(x, \tau)+B\left(u_{m-1}(x, \tau)\right)\right) d \tau
\end{aligned}
$$

And the following results are obtained:

$$
\begin{gathered}
u_{1}(x, t)=-4 h t \operatorname{sech} x, \\
v_{1}(x, t)=0, \\
u_{2}(x, t)=-4 h(h+n) t \operatorname{sech} x, \\
v_{2}(x, t)=-4 h^{2} t^{2} \operatorname{sech}^{3} x, \\
u_{3}(x, t)=-4 h(h+n)^{2} t \operatorname{sech} x+\frac{4}{3} h^{3} t^{3} \operatorname{sech}^{3} x,
\end{gathered}
$$

$u_{m}(x, t),(m=4,5, \ldots)$ can be calculated similarly. Then, the series solution expression by mq-HAM can be written in the following form:

$$
u(x, t ; n ; h) \cong U_{M}(x, t ; n ; h)=\sum_{i=0}^{M} u_{i}(x, t ; n ; h)\left(\frac{1}{n}\right)^{i} .
$$

Equation (39) is a family of approximation solutions to the problem (28) in terms of the convergence parameters $h$ and $n$. To find the valid region of $h$, the $h$-curves given by the 6th-order $n$ HAM (mq-HAM; $n=1)$ approximation and 


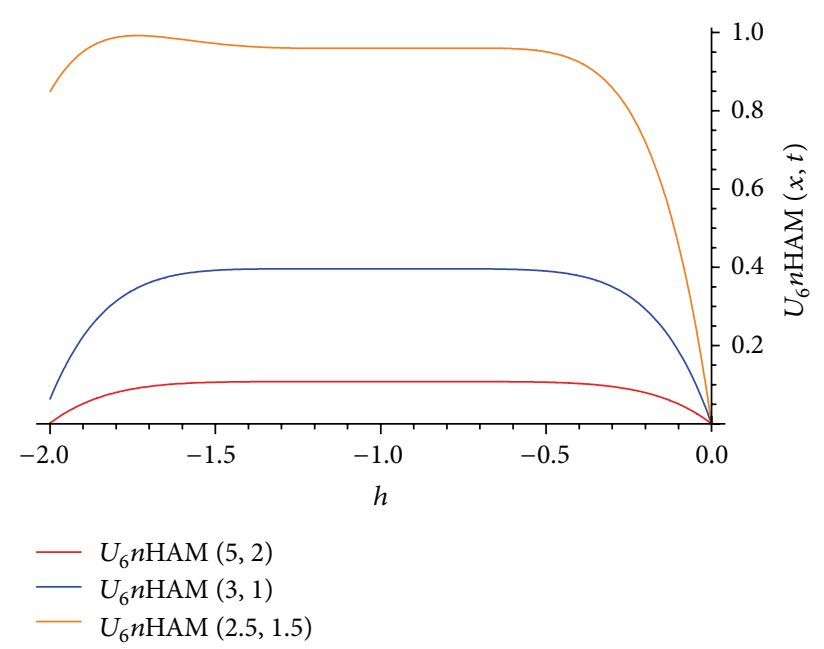

FIGURE 1 : $h$-curve for the $n$ HAM $(m q$-HAM; $n=1)$ approximation solution $U_{6}(x, t)$ of problem (28) at different values of $x$ and $t$.

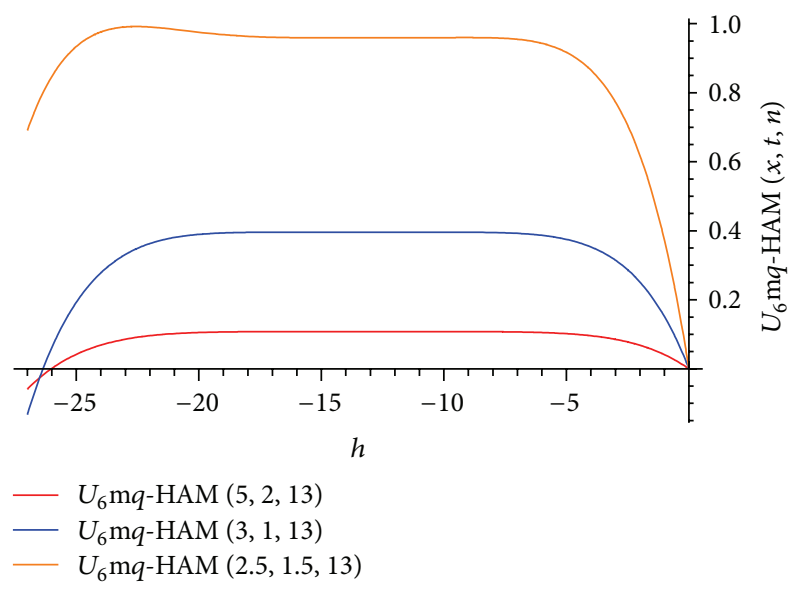

FIGURE 2: $h$-curve for the (mq-HAM; $n=13)$ approximation solution $U_{6}(x, t ; 13)$ of problem $(28)$ at different values of $x$ and $t$.

the 6th-order mq-HAM $(n=13)$ approximation at different values of $x, t$ are drawn in Figures 1 and 2, respectively, and these figures show the interval of $h$ in which the value of $U_{6}$ is constant at certain $x, t$, and $n$; we chose the horizontal line parallel to $x$-axis $(h)$ as a valid region which provides us with a simple way to adjust and control the convergence region. Figure 3 shows the comparison between $U_{6}$ of $n \mathrm{HAM}$ and $U_{6}$ of mq-HAM using different values of $n$ with the solution (30). The absolute errors of the 6th-order solutions $n$ HAM approximate and the 6th-order solutions mq-HAM approximate using different values of $n$ are shown in Figure 4. The results obtained by mq-HAM indicate that the speed of convergence for m $q$-HAM with $n>1$ is faster in comparison to $n=1(n \mathrm{HAM})$. The results show that the convergence region of series solutions obtained by $\mathrm{m} q$-HAM is increasing as $q$ is decreased as shown in Figures 3 and 4.

By increasing the number of iterations by mq-HAM, the series solution becomes more accurate, more efficient, and

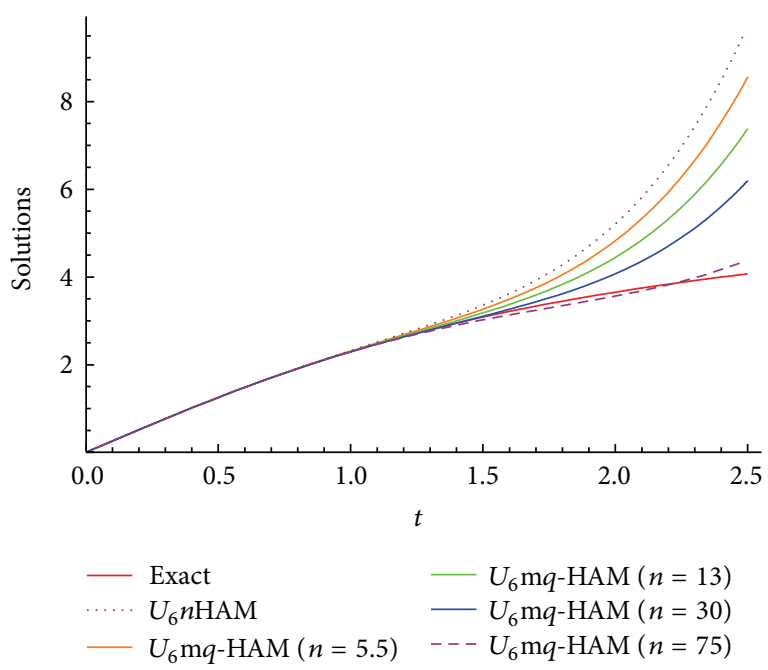

FIGURE 3: Comparison between $U_{6}$ of $n \mathrm{HAM}(\mathrm{m} q-\mathrm{HAM} ; n=1)$ and $U_{6}$ mq-HAM $(n=5.5,13,30,75)$ with exact solution of $(28)$ at $x=1$ with $(h=-1, h=-4.9, h=-10.8, h=-23.15, h=-49.25)$, respectively.
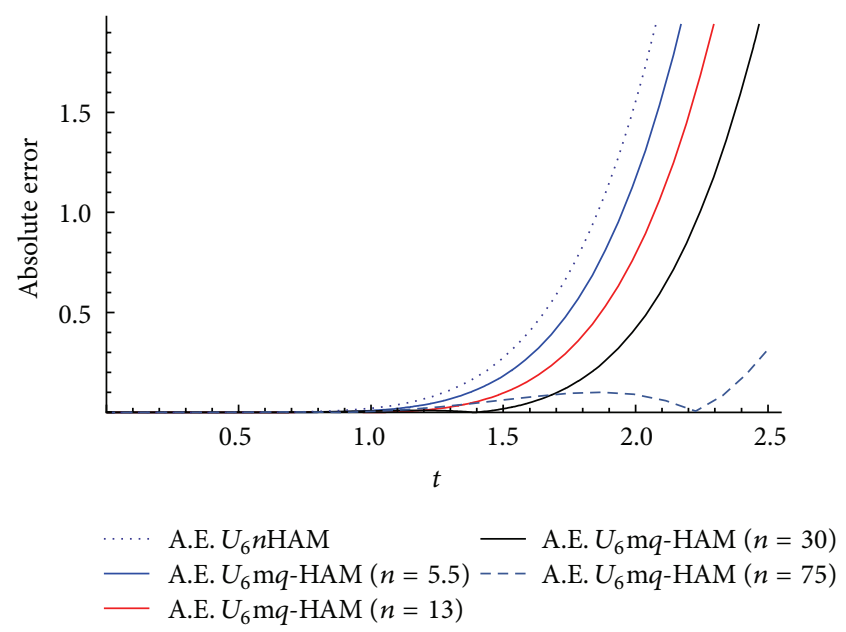

FIgURE 4: The absolute error of $U_{6}$ of $n \mathrm{HAM}$ (mq-HAM; $n=1$ ) and $U_{6}$ mq-HAM $(n=5.5,13,30,75)$ for problem $(28)$ at $x=1$ using $(h=-1, h=-4.9, h=-10.8, h=-23.15, h=-49.25)$, respectively.

the interval of $t$ (convergent region) increases as shown in Figures 5, 6, 7, and 8 .

Example 2. Consider the following Klein-Gordon equation:

$$
u_{t t}-u_{x x}+\frac{3}{4} u-\frac{3}{2} u^{3}=0,
$$

subject to the following initial conditions:

$$
\begin{gathered}
u(x, 0)=-\operatorname{sech} x, \\
u_{t}(x, 0)=\frac{1}{2} \operatorname{sech} x \tanh x .
\end{gathered}
$$




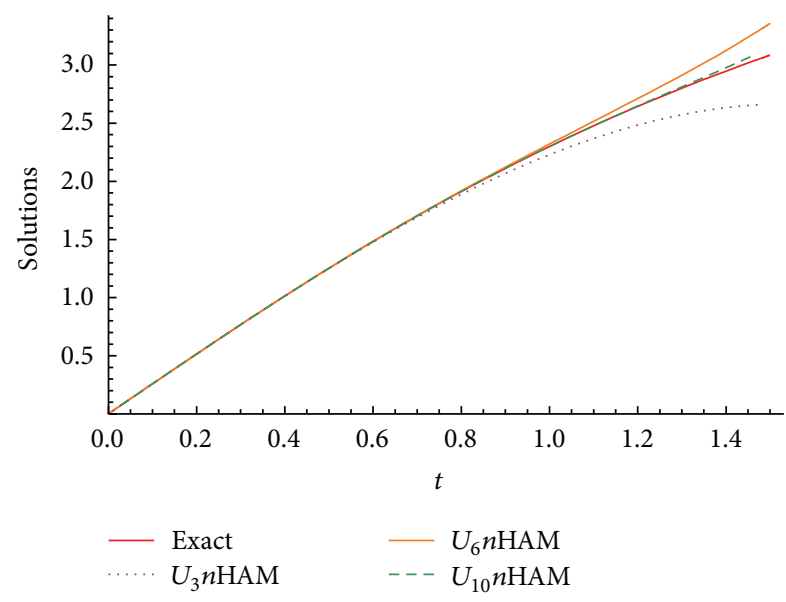

FIgURE 5: The comparison between the $U_{3}, U_{6}$, and $U_{10}$ of $n \mathrm{HAM}$ (mq-HAM; $n=1)$ and the exact solution of (28) at $h=-1$ and $x=1$.

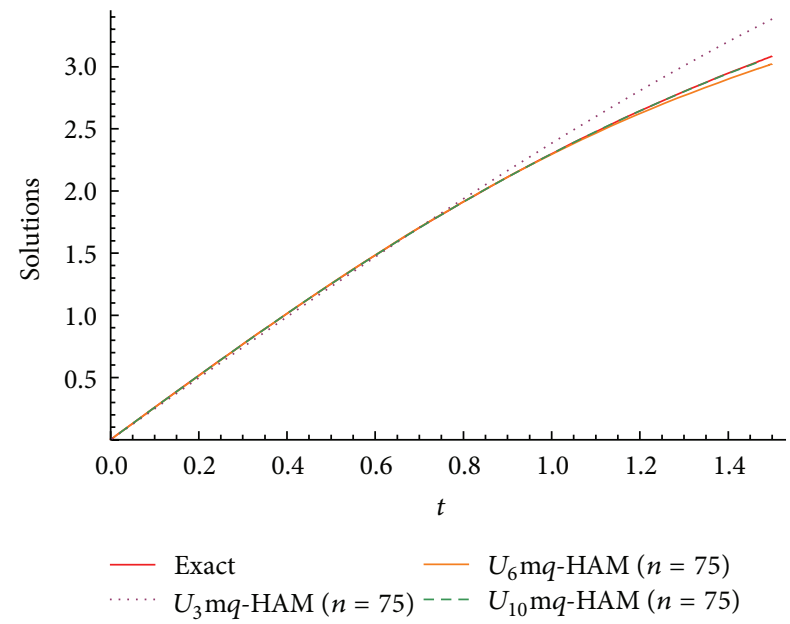

FIgURE 6: The comparison between the $U_{3}, U_{6}$, and $U_{10}$ of mq-HAM $(n=75)$ and the exact solution of $(28)$ at $h=-49.25$ and $x=1$.

The exact solution is

$$
u(x, t)=-\operatorname{sech}\left(x+\frac{t}{2}\right)
$$

In order to solve (40) by mq-HAM, we construct system of differential equations as follows:

$$
\begin{gathered}
u_{t}(x, t)=v(x, t), \\
v_{t}(x, t)=\frac{\partial^{2} u(x, t)}{\partial x^{2}}-\frac{3}{4} u+\frac{3}{2} u^{3},
\end{gathered}
$$

with the following initial approximations:

$$
u_{0}(x, t)=-\operatorname{sech} x, \quad v_{0}(x, t)=\frac{1}{2} \operatorname{sech} x \tanh x
$$

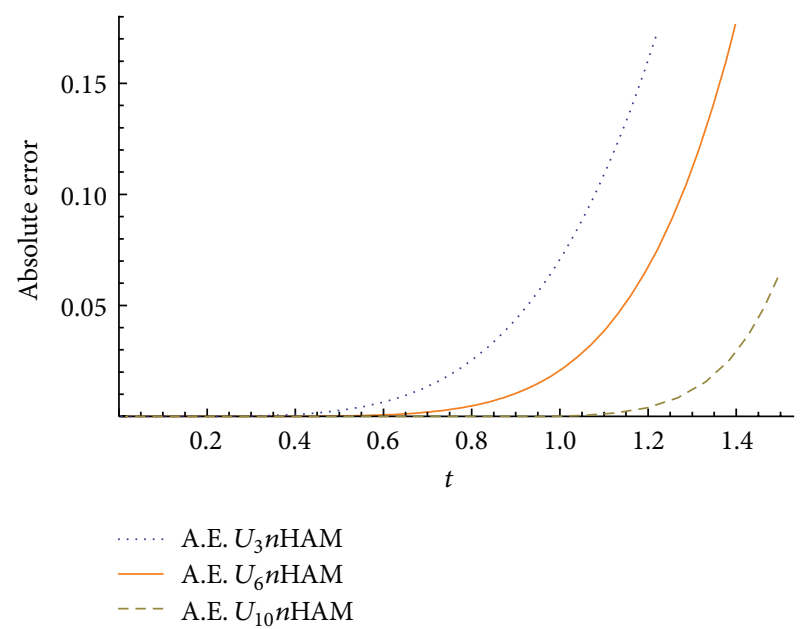

FIGURE 7: The comparison between the absolute error of $U_{3}, U_{6}$, and $U_{10}$ of $n$ HAM (mq-HAM; $\left.n=1\right)$ of (28) at $h=-1, x=1$, and $0 \leq t \leq 1.5$.
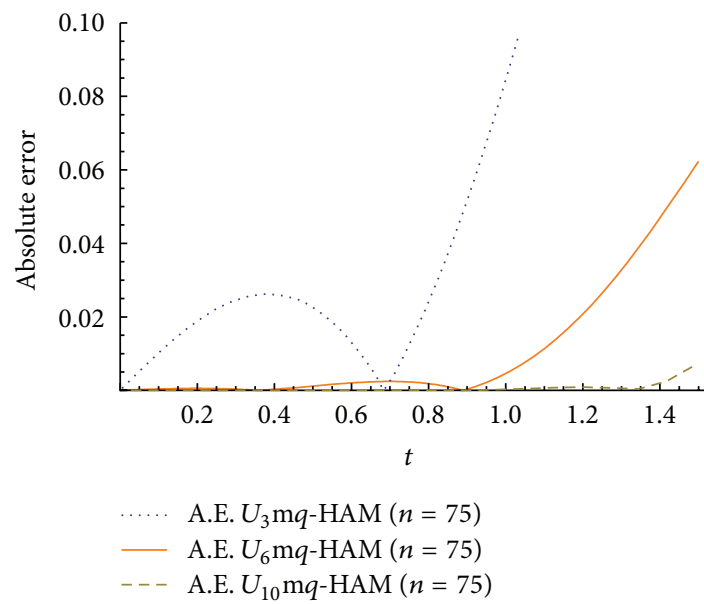

FIGURE 8: The comparison between the absolute error of $U_{3}, U_{6}$, and $U_{10}$ of mq-HAM $(n=75)$ of $(28)$ at $h=-49.25, x=1$, and $0 \leq t \leq$ 1.5 .

and the following auxiliary linear operators:

$$
\begin{gathered}
L u(x, t)=\frac{\partial u(x, t)}{\partial t}, \quad L v(x, t)=\frac{\partial v(x, t)}{\partial t}, \\
A u_{m-1}(x, t)=-\frac{\partial^{2} u_{m-1}(x, t)}{\partial x^{2}}+\frac{3}{4} u_{m-1}(x, t), \\
B \overrightarrow{u_{m-1}}(x, t)=-\frac{3}{2} \sum_{j=0}^{m-1} u_{m-1-j} \sum_{i=0}^{j} u_{i} u_{j-i} .
\end{gathered}
$$

From (25) and (27), we obtain

$$
\begin{gathered}
u_{1}(x, t)=h \int_{0}^{t}\left(-v_{0}(x, \tau)\right) d \tau \\
v_{1}(x, t)=h \int_{0}^{t}\left(-\frac{\partial^{2} u_{0}}{\partial x^{2}}+\frac{3}{4} u_{0}-\frac{3}{2} u_{0}^{3}\right) d \tau .
\end{gathered}
$$




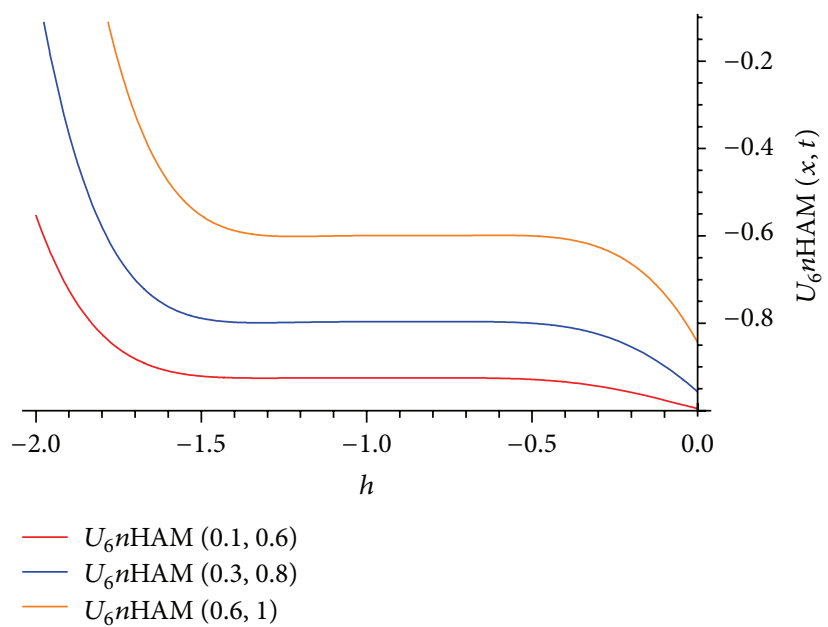

FIGURE 9: $h$-curve for the $n$ HAM $($ m $q$-HAM; $n=1)$ approximation solution $U_{6}(x, t)$ of problem (40) at different values of $x$ and $t$.

For $m \geq 2$, we get

$$
\begin{aligned}
& u_{m}(x, t)=(n+h) u_{m-1}(x, t)+h \int_{0}^{t}\left(-v_{m-1}(x, \tau)\right) d \tau, v_{m}(x, t)=(n+h) v_{m-1}(x, t) \\
&+h \int_{0}^{t}\left(-\frac{\partial^{2} u_{m-1}(x, t)}{\partial x^{2}}+\frac{3}{4} u_{m-1}(x, t)\right. \\
&\left.-\frac{3}{2} \sum_{j=0}^{m-1} u_{m-1-j} \sum_{i=0}^{j} u_{i} u_{j-i}\right) d \tau .
\end{aligned}
$$

The following results are obtained:

$$
\begin{gathered}
u_{1}(x, t)=-\frac{1}{2} h t \operatorname{sech} x \tanh x, \\
v_{1}(x, t)=h t\left(-\frac{3 \operatorname{sech} x}{4}+\frac{\operatorname{sech}^{3} x}{2}+\operatorname{sech} x \tanh ^{2} x\right), \\
u_{2}(x, t)=h\left(\frac{3}{16} h t^{2} \operatorname{sech}^{3} x-\frac{1}{16} h t^{2} \cosh (2 x) \operatorname{sech}^{3} x\right) \\
-\frac{1}{2} h(h+n) t \operatorname{sech} x \tanh x,
\end{gathered}
$$

$u_{m}(x, t),(m=3,4, \ldots)$ can be calculated similarly. Then, the series solution expression by $\mathrm{m} q$-HAM can be written in the following form:

$$
u(x, t ; n ; h) \cong U_{M}(x, t ; n ; h)=\sum_{i=0}^{M} u_{i}(x, t ; n ; h)\left(\frac{1}{n}\right)^{i} .
$$

Equation (49) is a family of approximation solutions to the problem (40) in terms of the convergence parameters $h$ and $n$. To find the valid region of $h$, the $h$-curves given by the 6th-order $n$ HAM (mq-HAM; $n=1)$ approximation and

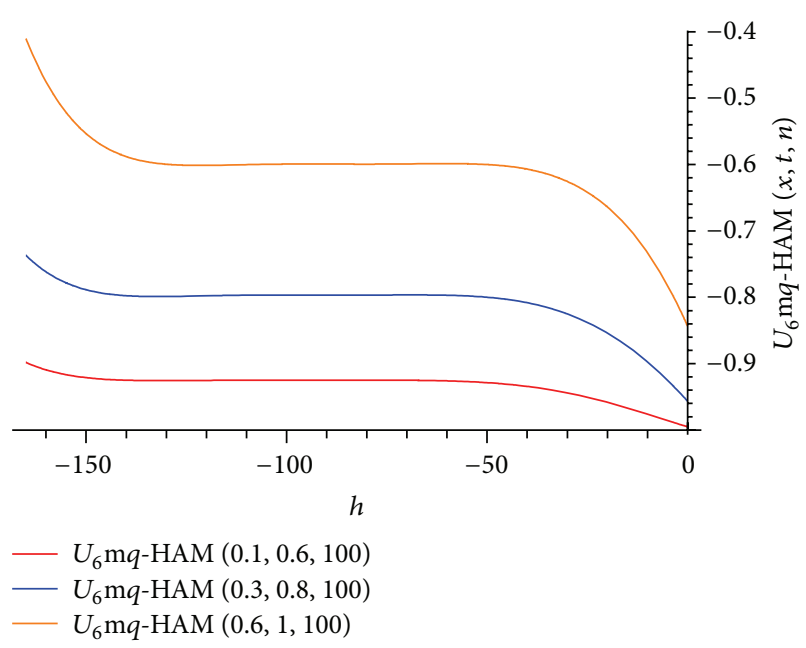

Figure 10: $h$-curve for the mq-HAM $(n=100)$ approximation solution $U_{6}(x, t ; 100)$ of problem $(40)$ at different values of $x$ and $t$.

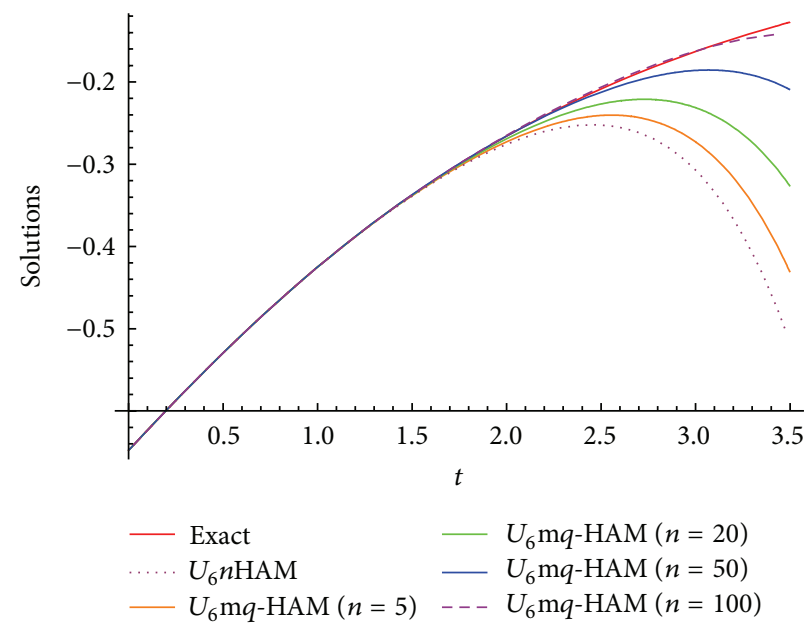

Figure 11: Comparison between $U_{6}$ of $n$ HAM (mq-HAM; $n=1$ ) and $U_{6}$ of mq-HAM $(n=5,20,50,100)$ with exact solution of $(40)$ at $x=1$ with $(h=-1, h=-4.85, h=-18.55, h=-43.11, h=$ $-79.5)$, respectively.

the 6th-order mq-HAM $(n=100)$ approximation at different values of $x, t$ are drawn in Figures 9 and 10; these figures show the interval of $h$ in which the value of $U_{6}$ is constant at certain $x, t$, and $n$; we chose the horizontal line parallel to $x$-axis $(h)$ as a valid region which provides us with a simple way to adjust and control the convergence region. Figure 11 shows the comparison between $U_{6}$ of $n$ HAM and $U_{6}$ of mq-HAM using different values of $n$ with the solution (42). The absolute errors of the 6th-order solutions $n$ HAM approximate and the 6thorder solutions mq-HAM approximate using different values of $n$ are shown in Figure 12. The results obtained by $\mathrm{m} q-$ HAM indicate that the speed of convergence for mq-HAM with $n>1$ is faster in comparison to $n=1$ ( $n$ HAM). The results show that the convergence region of series solutions 


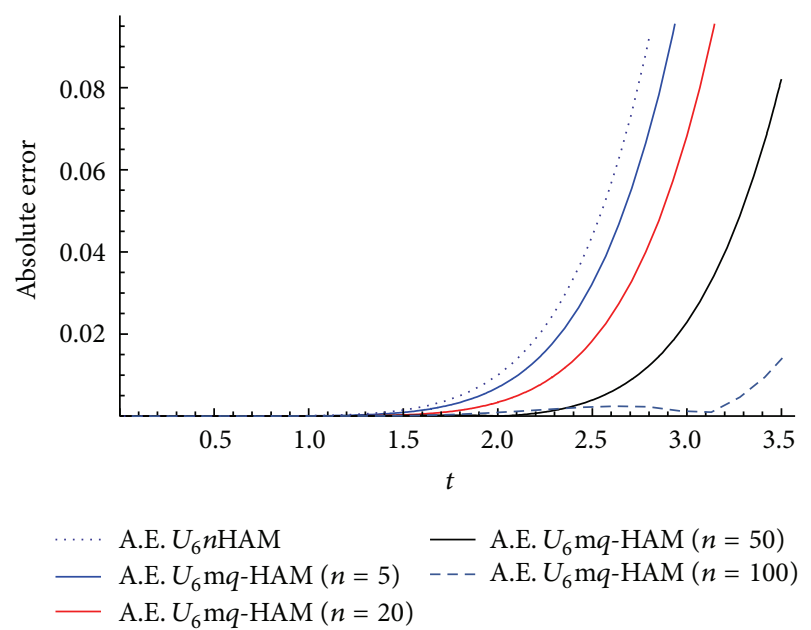

FIgURe 12: The absolute error of $U_{6}$ of $n$ HAM (mq-HAM; $n=1$ ) and $U_{6}$ of mq-HAM $(n=5,20,50,100)$ for problem $(40)$ at $x=1$ using $(h=-1, h=-4.85, h=-18.55, h=-43.11, h=-79.5)$, respectively.

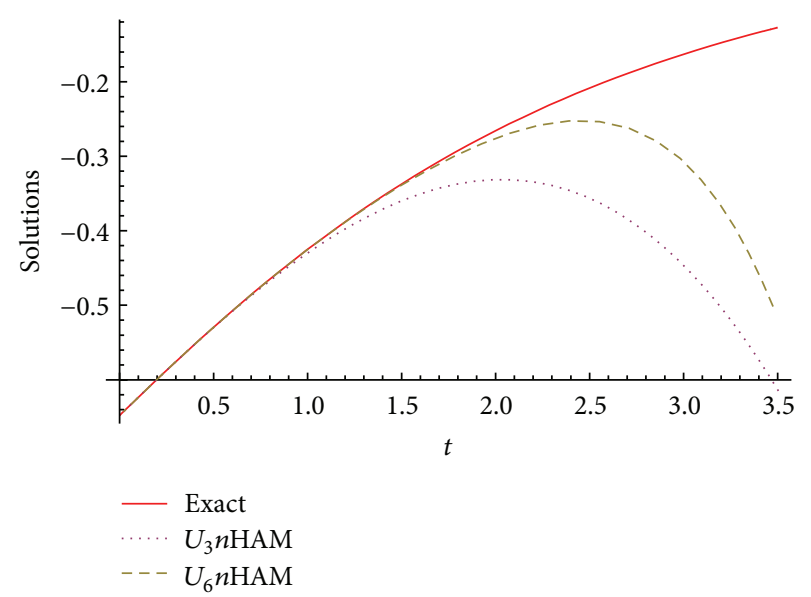

Figure 13: The comparison between the $U_{3}, U_{6}$ of $n$ HAM (mqHAM; $n=1)$, and the exact solution of $(40)$ at $h=-1$ and $x=1$.

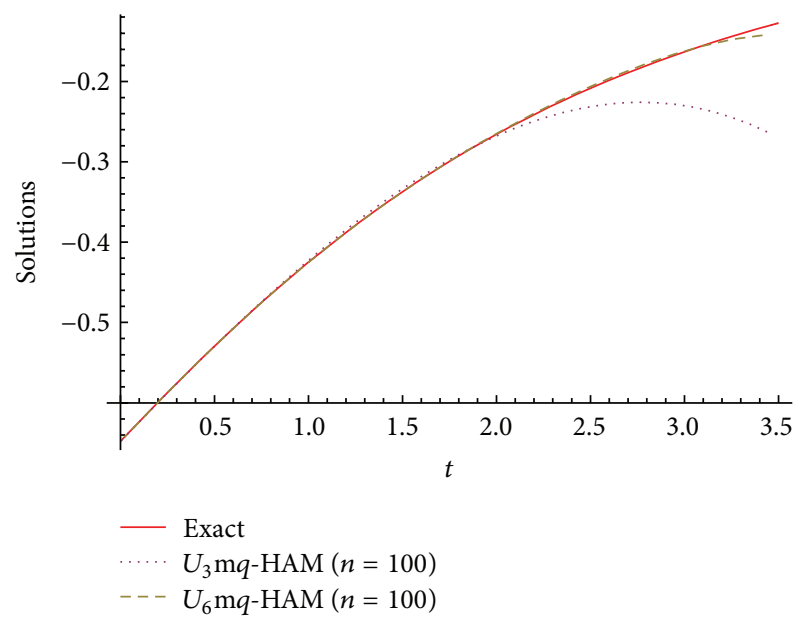

FIGURE 14: The comparison between the $U_{3}, U_{6}$ of mq-HAM $(n=$ $100)$, and the exact solution of (40) at $h=-79.5$ and $x=1$.

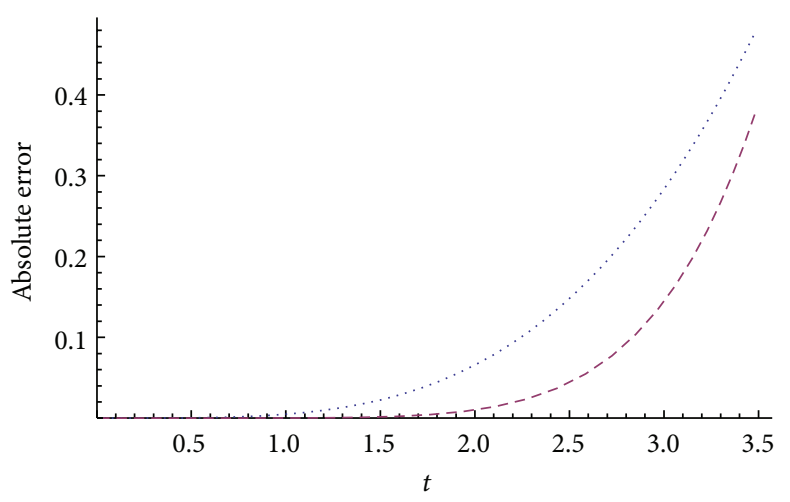

A.E. $U_{3} n \mathrm{HAM}$
--- A.E. $U_{6} n \mathrm{HAM}$

FIGURE 15: The comparison between the absolute error of $U_{3}$ and $U_{6}$ of $n$ HAM (mq-HAM; $\left.n=1\right)$ of (40) at $h=-1, x=1$, and $0 \leq t \leq 3.5$.

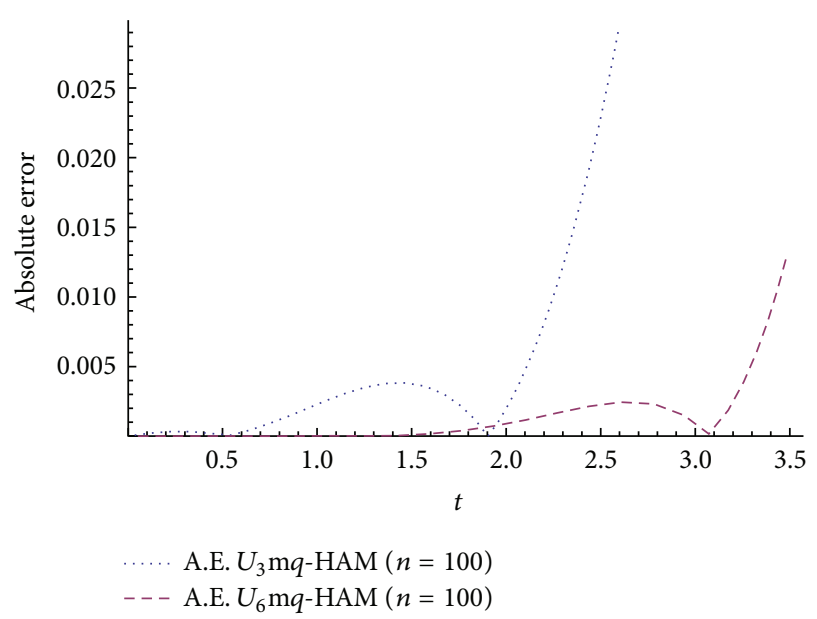

FIGURE 16: The comparison between the absolute error of $U_{3}$ and $U_{6}$ of $\operatorname{ma}-\operatorname{HAM}(n=100)$ of $(40)$ at $h=-79.5, x=1$, and $0 \leq t \leq 3.5$.

obtained by $\mathrm{m} q$-HAM is increasing as $q$ is decreased as shown in Figures 11 and 12.

By increasing the number of iterations by mq-HAM, the series solution becomes more accurate, more efficient, and the interval of $t$ (convergent region) increases as shown in Figures 13, 14, 15, and 16.

Figure 17 shows that the convergence of the series solutions obtained by the 3rd-order mq-HAM $(n=100)$ is faster than that of the series solutions obtained by the 6 th order $n$ HAM. This fact shows the importance of the convergence parameters $n$ in the m $q$-HAM.

\section{Conclusion}

In this paper, a modified $q$-homotopy analysis method was proposed (mq-HAM). This method provides an approximate solution by rewriting the $n$ th-order nonlinear differential equations in the form of system of $n$ first-order differential equations. The solution of these $n$ differential equations is 


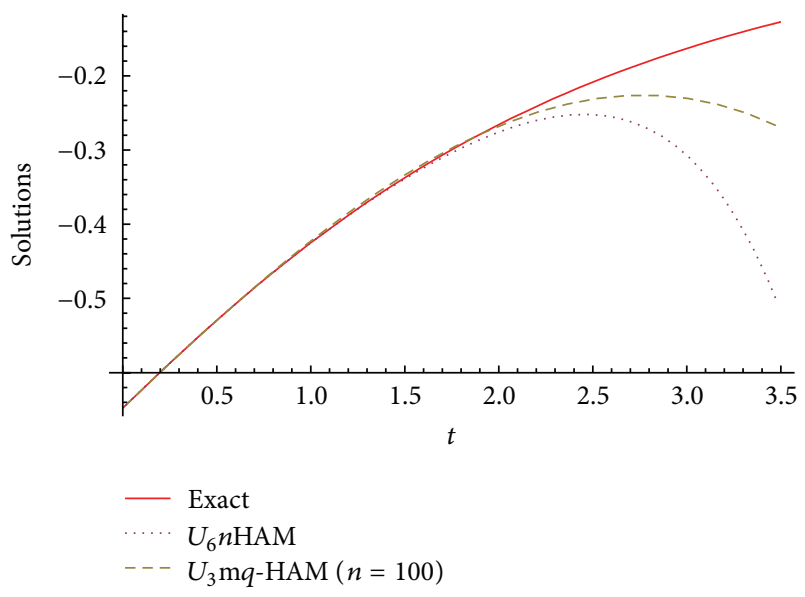

FIGURE 17: The comparison between the $U_{3}$ of mq-HAM $(n=100)$, $U_{6}$ of $n$ HAM (mq-HAM; $n=1$ ), and the exact solution of $(40)$ at $(h=-79.5, h=-1)$ and $x=1$.

obtained as a power series solution, which converges to a closed form solution. The mq-HAM contains two auxiliary parameters $n$ and $h$ such that the case of $n=1$ (mq-HAM; $n=$ 1 ); the $n$ HAM which is proposed in $[21,22]$ can be reached. In general, it was noticed from the illustrative examples that the convergence of mq-HAM is faster than that of $n$ HAM.

\section{References}

[1] S. J. Liao, The proposed homotopy analysis technique for the solution of nonlinear problems [Ph.D. thesis], Shanghai Jiao Tong University, 1992.

[2] S. Abbasbandy, "The application of homotopy analysis method to nonlinear equations arising in heat transfer," Physics Letters A, vol. 360, no. 1, pp. 109-113, 2006.

[3] S. Abbasbandy and A. Shirzadi, "Homotopy analysis method for multiple solutions of the fractional Sturm-Liouville problems," Numerical Algorithms, vol. 54, no. 4, pp. 521-532, 2010.

[4] S. Abbasbandy, M. Ashtiani, and E. Babolian, "Analytic solution of the Sharma-Tasso-Olver equation by homotopy analysis method," Zeitschrift fur Naturforschung A, vol. 65, no. 4, pp. 285290, 2010.

[5] A. K. Alomari, M. S. M. Noorani, and R. Nazar, "Explicit series solutions of some linear and nonlinear Schrodinger equations via the homotopy analysis method," Communications in Nonlinear Science and Numerical Simulation, vol. 14, no. 4, pp. 1196-1207, 2009.

[6] M. Ayub, A. Rasheed, and T. Hayat, "Exact flow of a third grade fluid past a porous plate using homotopy analysis method," International Journal of Engineering Science, vol. 41, no. 18, pp. 2091-2103, 2003.

[7] A. Sami Bataineh, M. S. M. Noorani, and I. Hashim, "Solving systems of ODEs by homotopy analysis method," Communications in Nonlinear Science and Numerical Simulation, vol. 13, no. 10, pp. 2060-2070, 2008.

[8] A. S. Bataineh, M. S. M. Noorani, and I. Hashim, "Homotopy analysis method for singular IVPs of Emden-Fowler type," Communications in Nonlinear Science and Numerical Simulation, vol. 14, no. 4, pp. 1121-1131, 2009.
[9] T. Hayat, M. Khan, and S. Asghar, "Homotopy analysis of MHD flows of an Oldroyd 8-constant fluid," Acta Mechanica, vol. 168, no. 3-4, pp. 213-232, 2004.

[10] S. J. Liao, Beyond Perturbation: Introduction to the Homotopy Analysis Method, Chapman \& Hall, Boca Raton, Fla, USA, 2004.

[11] S. J. Liao, "An approximate solution technique not depending on small parameters: a special example," International Journal of Non-Linear Mechanics, vol. 30, no. 3, pp. 371-380, 1995.

[12] S. Liao, "On the homotopy analysis method for nonlinear problems," Applied Mathematics and Computation, vol. 147, no. 2, pp. 499-513, 2004.

[13] S. Liao, "Comparison between the homotopy analysis method and homotopy perturbation method," Applied Mathematics and Computation, vol. 169, no. 2, pp. 1186-1194, 2005.

[14] A. Molabahrami and F. Khani, "The homotopy analysis method to solve the Burgers-Huxley equation," Nonlinear Analysis: Real World Applications, vol. 10, no. 2, pp. 589-600, 2009.

[15] S. Nadeem and N. S. Akbar, "Peristaltic flow of Sisko fluid in a uniform inclined tube," Acta Mechanica Sinica, vol. 26, no. 5, pp. 675-683, 2010.

[16] S. Nadeem and N. S. Akbar, "Influence of heat transfer on a peristaltic flow of Johnson Segalman fluid in a non uniform tube," International Communications in Heat and Mass Transfer, vol. 36, no. 10, pp. 1050-1059, 2009.

[17] Y. Tan and S. Abbasbandy, "Homotopy analysis method for quadratic Riccati differential equation," Communications in Nonlinear Science and Numerical Simulation, vol. 13, no. 3, pp. 539-546, 2008.

[18] A. Sami Bataineh, M. S. M. Noorani, and I. Hashim, "Approximate solutions of singular two-point BVPs by modified homotopy analysis method," Physics Letters A, vol. 372, pp. 40624066, 2008.

[19] A. Sami Bataineh, M. S. M. Noorani, and I. Hashim, "On a new reliable modification of homotopy analysis method," Communications in Nonlinear Science and Numerical Simulation, vol. 14, no. 2, pp. 409-423, 2009.

[20] A. S. Bataineh, M. S. M. Noorani, and I. Hashim, "Modified homotopy analysis method for solving systems of second-order BVPs," Communications in Nonlinear Science and Numerical Simulation, vol. 14, no. 2, pp. 430-442, 2009.

[21] H. N. Hassan and M. A. El-Tawil, "A new technique of using homotopy analysis method for solving high-order nonlinear differential equations," Mathematical Methods in the Applied Sciences, vol. 34, no. 6, pp. 728-742, 2011.

[22] H. N. Hassan and M. A. El-Tawil, "A new technique of using homotopy analysis method for second order nonlinear differential equations," Applied Mathematics and Computation, vol. 219, no. 2, pp. 708-728, 2012.

[23] M. A. El-Tawil and S. N. Huseen, "The q-homotopy analysis method (q-HAM)," International Journal of Applied Mathematics and Mechanics, vol. 8, no. 15, pp. 51-75, 2012.

[24] M. A. El-Tawil and S. N. Huseen, "On convergence of the qhomotopy analysis method," International Journal of Contemporary Mathematical Sciences, vol. 8, no. 10, pp. 481-497, 2013. 


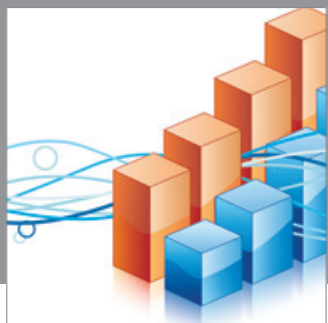

Advances in

Operations Research

mansans

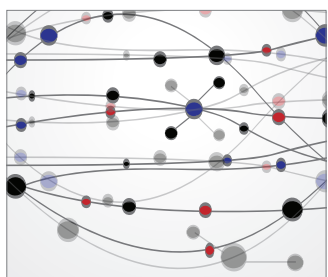

The Scientific World Journal
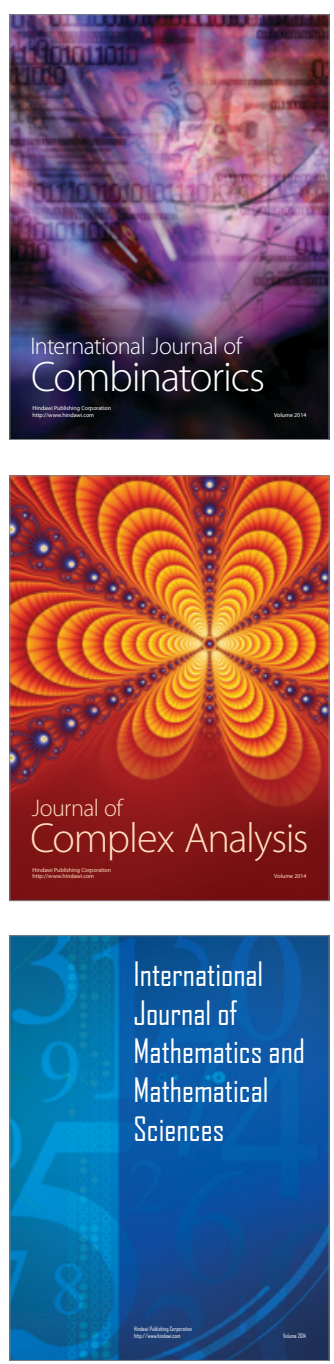
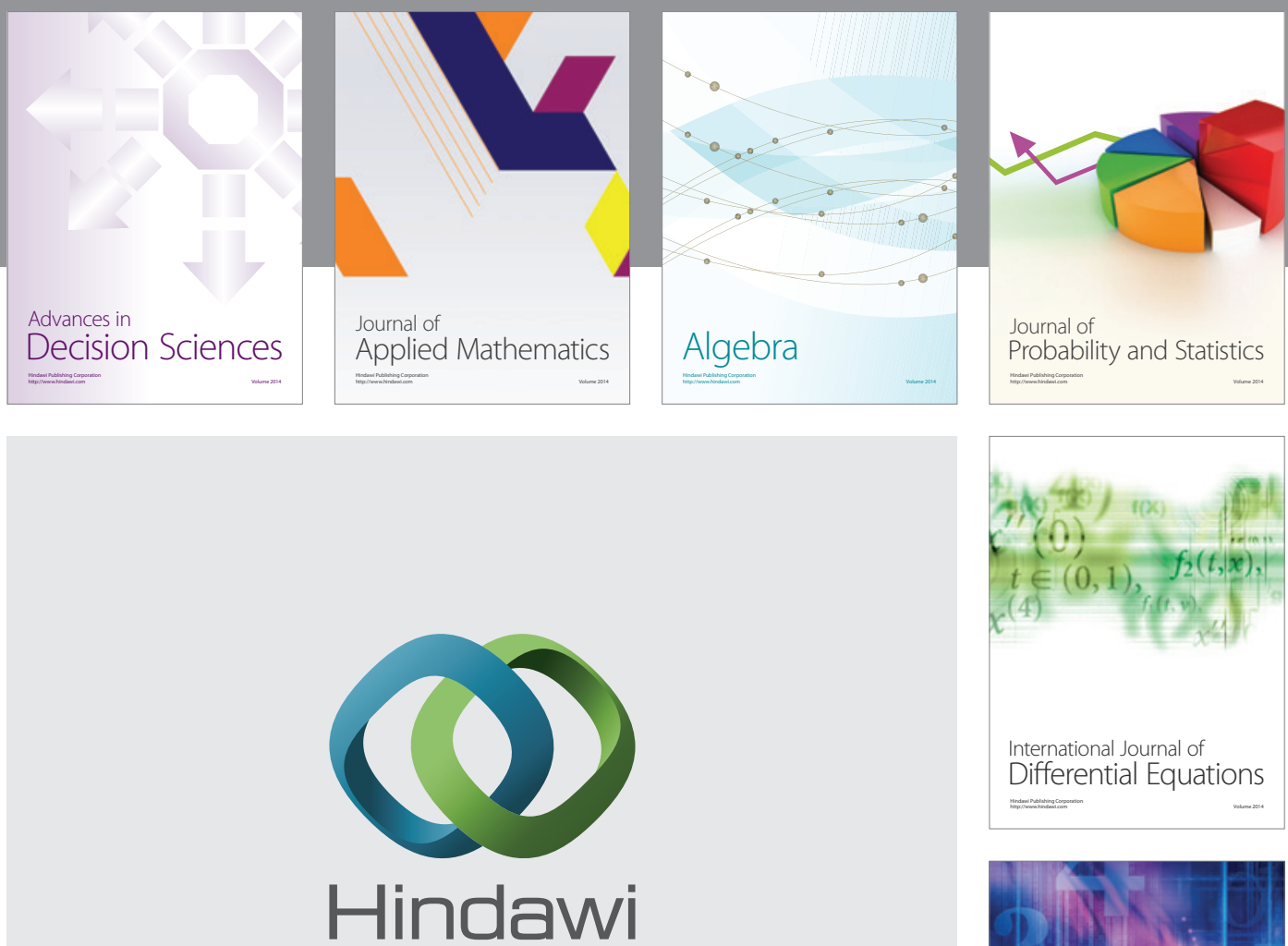

Submit your manuscripts at http://www.hindawi.com
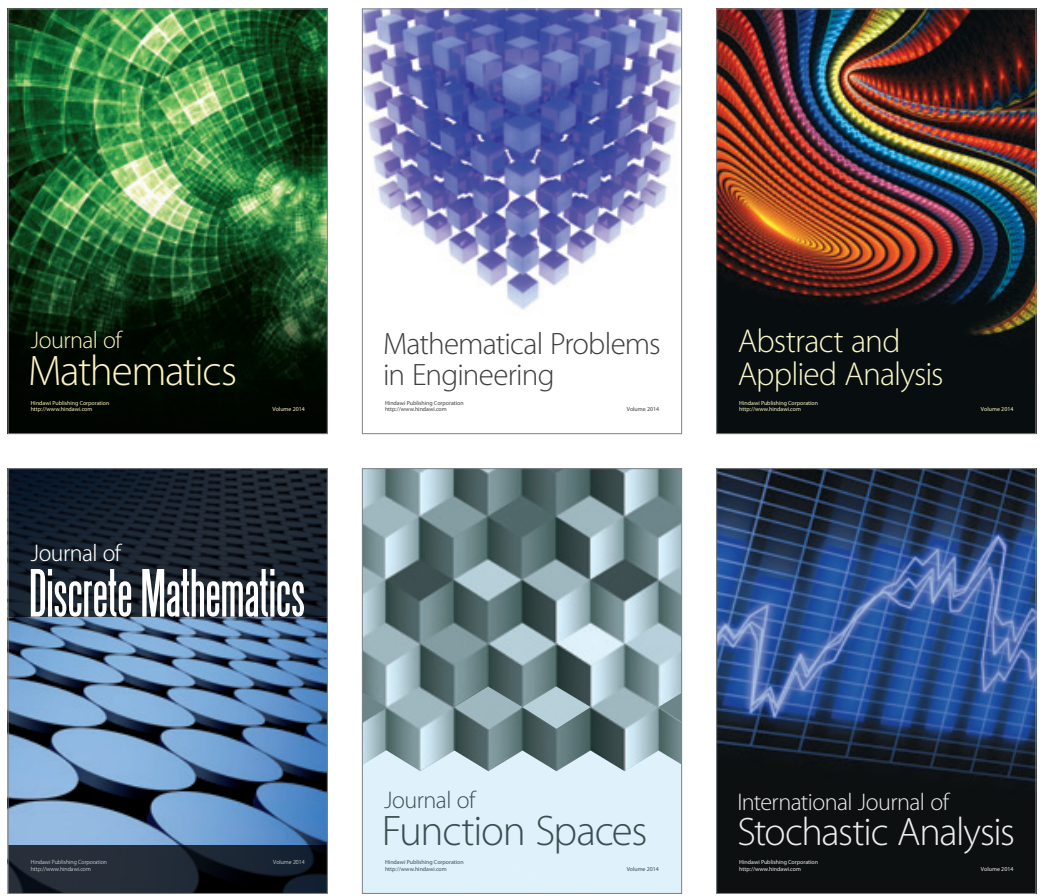

Journal of

Function Spaces

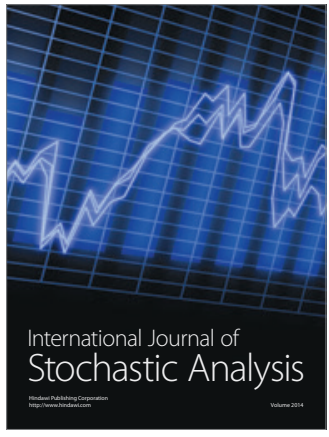

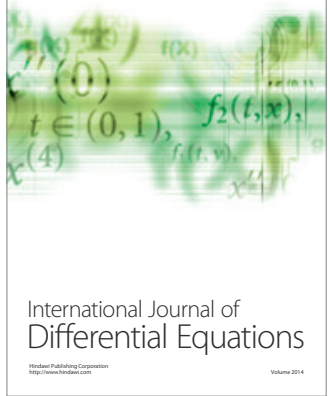
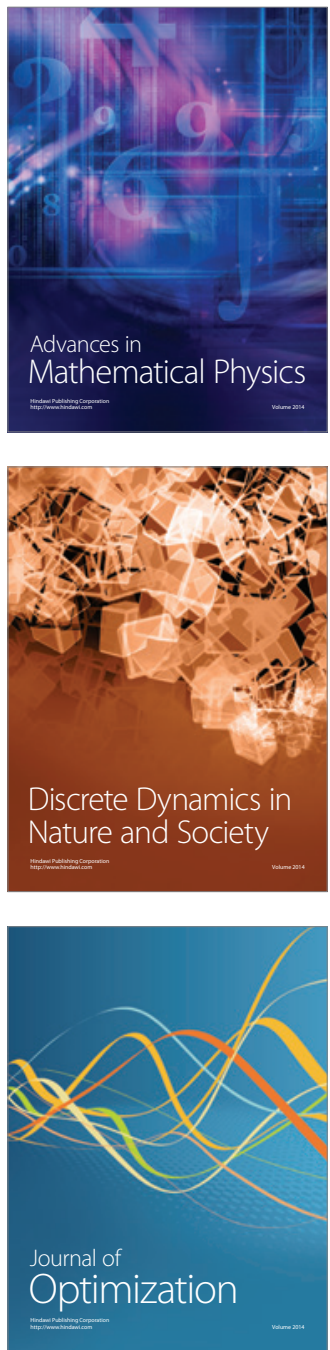